Вісник Дніпропетровського університету. Серія: геологія, географія. 2016. 24 (1), 107-111.

Vìsnik Dnìpropetrovs'kogo unìversitetu. Serîâ geologîâ, geographîâ

Dnipropetrovsk University Bulletin. Series geology, geography. 2016, 24 (1), 107-111.

doi: $10.15421 / 111616$

http://geology-dnu.dp.ua

УДК 624.131:(477)

\title{
Возможности прогноза активности оползневых явлений по результатам индуктивного моделирования и дистанционных методов исследований
}

\author{
Т. П. Мокрицкая, М. Л. Ярощук, Д.А. Довганенко \\ Днепропетровский национальный университет имени Олеся Гончара, Днепропетровск, Украина, \\ e-mail: mtatjana@bk.ru
}

Рассмотрены возможности анализа и прогноза оползневой активности по материалам дистанционного зондирования с привлечением методов индуктивного моделирования. Использование метода группового учета аргументов показало, что возможно создание математических моделей связи значений приходящего на сенсор излучения и морфологических признаков оползневых тел, находящихся в фазе активного развития. Доказано влияние на активность развития оползневого процесса фаз солнечной активности 11-летнего ритма, в том числе, на застроенных территориях. Построенные модели могут быть применены для прогноза активности оползневого процесса.

Ключевые слова: оползень, дистанционное зондирование, метод группового учета аргументов, прогноз

\section{The possibility of predicting the activity of landslides as a result of inductive modeling and remote research methods}

\author{
T. Mokritskaya, M. Yaroschuk, D. Dovganenko \\ Oles Honchar Dnepropetrovsk National University, Dnepropetrovsk, Ukraine, e-mail: mtatjana@bk.ru
}

The object of study: Landslide processes developing in the Dnepropetrovskcity. Objective: to substantiate the accuracy of the existing classification of landslides in the Dnepropetrovsk city in order to develop methods of prediction landslide activity of remote research. Research Method: cartographic modeling, mathematical modeling, which includes the primary statistic, correlation, clustering, regression types of stochastic analysis and inductive modeling (group method of data). It was found that the solution of the research and prediction of landslides activation is impossible without an integrated approach to the study and analysis of landslide processes. The best results in the solution of this problem can be obtained by the monitoring of geological environment with using remote sensing (RS). In this work, the forecast of landslide activity methods of inductive modeling (group method of data) on the basis of a joint analysis results of monitoring the geological environment of Dnepropetrovsk, Dneprodzerzhinsk agglomeration (1983 - 2006. «Ukryuzhgeologiya») and are available free of remote sensing satellites LANLASAT 5 and LANLASAT .

Keywords: landslide, remote sensing, group method of data, forecast

Введение. Применение дистанционных методов исследований для прогноза вероятности возникновения оползневых явлений, карстово-суффозионных провалов и других опасностей широко используется в практике инженерно-геологических (Pardeshi, Autade, Pardeshi, 2013; Das;Shljahova, Povh, 2010) и эколого-геологических исследований(Montoya-Montes, Rodríguez-Santalla, Sánchez-García, Alcántara-Carriós, Martín-Velázquez, Gómez-Ortiz, Martín-Crespo, 2012; Patel, Sahoo, Singh, 2013; Caers, 2011). В работе (Pardeshi, Autade, Pardeshi, 2013) выполнен обзор современных методов обработки материалов дистанционного зондирования, применяющихся на следующих этапах создания прогнозных моделей, геодинамического риска: анализ материалов, сортировка данных, распознавание явлений. Преобладание методов вероятностного моделирования объясняется многофакторностью опасных геологических процессов, что выражается в некоторой неопределенности свойств среды и данных о ней и приводит к необходимости выбора стохастических и иных методов. Методика картирования оползне- 
вой активности была разработана и апробирована в существующих региональных инженерно-геологических условиях в работах (Emel'janova, 1971; Gulakjan, Kjuncel', Postoev, 1977; Koposov, 2012). Использование материалов дистанционных исследований лежит в основе анализа и прогноза опасных геодинамических явлений на территории Украины (Groisman, Lyalko, 2012). Привлечение методов индуктивного моделирования, в частности, метода группового учета аргументов,к анализу и прогнозу оползневой активности по материалам наземного мониторинга и дистанционного зондирования позволяет выполнить прогноз оползневой активности для явлений определенного типа.

Методы исследований. В данной работе выполнен прогноз оползневой активности методами индуктивного моделирования (метод группового учета аргументов) на основании совместного анализа результатов мониторинга геологической среды Днепропетровско-Днепродзержинской агломерации(1983 - 2006 гг., Укрюжгеология) и находящихся в свободном доступе материалов дистанционного зондирования спутников LANL $\Lambda$ SAT 5 и LANL $\Lambda$ SAT 7 $[14,15]$. Режимные наземные наблюдения за состоянием оползневых тел выполнялись на территории г. Днепропетровск на протяжении 1983-1995 гг., в дальнейшем выполнялись нерегулярные наблюдения. Общее количество оползневых тел составило 133. Материалы мониторинга оползневых тел переданы авторам для научной обработки безвозмездно. Привлечено авторское программное обеспечение (Mokrickaja, Korjashkina, 2013) триал - версия программы STATISTICA [14].

Алгоритм исследований можно представить в виде последовательности операций: создание баз данных мониторинга и материалов дистанционного зондирования, анализ данных с целью классификации оползневых процессов и явлений; описание связей между вероятными факторами, отбор влияющих факторов, краткосрочный прогноз.

Для выполнения исследований были созданы две независимые базы данных о результатах наземного мониторинга оползневых процессов и о значениях приходящего на сенсор излучения $\left(\boldsymbol{L} \lambda, \frac{\mathrm{BT}}{\mathrm{M}^{2} \cdot \text { ster } \mu \mathrm{M}}\right)$ в зонах активизации оползневых процессов. Формирование выборок приходящего на сенсор излучения $\boldsymbol{L} \boldsymbol{\lambda}$ был выполнено с помощью программы ENVI (Mokrickaja, Korjashkina, 2013).Даты определений $\boldsymbol{L} \boldsymbol{\lambda}$ образуютнерегулярныйи короткий ряд: 18 мая 1988 года, 18 февраля 1990 года, 20 февраля 2003 года, 23 апреля 2003 года, 26 июня 2003 года, 29 августа
2003 года, 25 августа 2006 года, 26 июня 2009 года, 22 января 2010 года, 25 марта 2010 года, 24 января 2011 года. Данные за 2003 год в первом приближении позволяют выполнить анализ связи между значениями приходящего на сенсор излучения $\boldsymbol{L} \boldsymbol{\lambda}$ на протяжении неполного годового ритма колебаний уровня подземных вод. Весь ряд имеет длительность 22 года, поэтому можно выполнить анализ влияния 11-летнего ритма солнечной активности на активность оползневых процессов. Так как в мае 1996 года начался 23 цикл солнечной активности, а в январе 2009 года - 24-й цикл, влияние могло проявиться в период подъема солнечной активности (Shljahova, Povh, 2010). По материалам (Koposov, 2012), активность на протяжении 23-го (нечетного) цикла должна возрастать, а на протяжении 24-го (четного) цикла солнечной активности - падать.

Изложение основного материала. Статистический анализ спектральных характеристик поверхности во всех точках, координаты которых соответствуют зонам активизации оползневого процесса различной интенсивности, показал, что вариативность значений достаточно резко изменяется. Увеличение вариативности сопровождается увеличением средних значений, асимметричность сохраняется, что указывает на временные особенности развития процесса вне зависимости от морфологии оползневых тел. Однородность распределений спектральных характеристик поверхности была проанализирована как за весь период наблюдений, так и в выбранные моменты. Аномальные значения эксцесса и асимметрии получены для моментов времени, близких к датам смены 11-летнего цикла (рис.). Рост асимметрии и эксцесса является признаком закономерных изменений приходящего на сенсор излучения, сопровождающихся формированием группировок.

Дальнейший анализ выполнялся выборочно, для зон развития активных оползневых тел и оплывин различной морфологии - циркообразных, фронтальных, ложкообразных, всего 131 точка наблюдений. Связи между описанными в ходе мониторинга количественными признаками формы оползневых тел, условиями их развития и значениями приходящего на сенсор излучения были изучены статистически. В качестве переменных, описывающих морфологию тел, выбраны длина, ширина, площадь оползневого тела, высота стенки отрыва. Длина и высота склона характеризуют условия развития оползневого процесса. Результаты корреляционного и регрессионного анализа приведены в таблицах 1 и 2.

Анализ результатов статического анализа показывает, что в качестве факторов приходящего на 


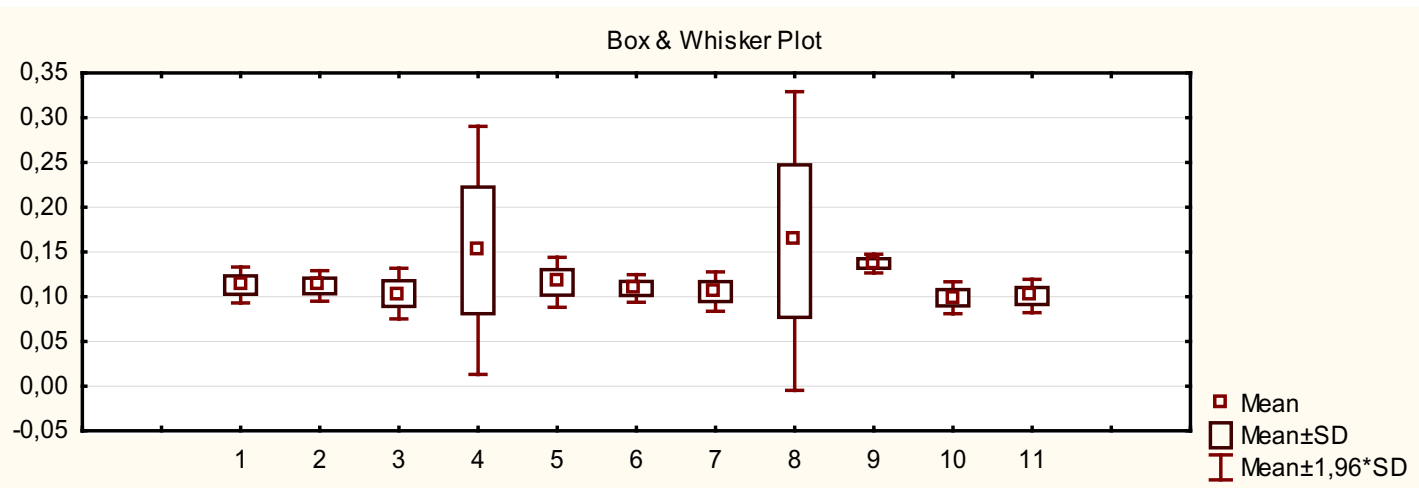

Рис. Неоднородность выборочных значений приходящего на сенсор излучения в зонах оползневых тел.

Примечания:

1.По оси ординат приведены значения $L \lambda, \frac{\text { Вт }}{\mathrm{M}^{2} \cdot \text { ster } \cdot \mu \mathrm{M}}$.

2.По оси аргументов указаны даты: $1-18.05 .1988,2$ - 18.02.1990, 3 - 20.02.2003, 4-23.04.2003,

5-26.06.2003, 6-29.08.2003, 7-25.08.2006, 8-26.06.2009, 9-22.01.2010, 10-25.03.2010, 11-24.01.2011

сенсор излучения выступаютвысота склона и параметры оползневого тела в период максимального

рованной составляющей в зависимость изменчивы (таблица 3). В качестве детерминированных перемен-

Статистические модели связи приходящего на сенсор излученияна участках активных оползневых тел и их Таблица 1 морфологических характеристик

\begin{tabular}{|c|c|c|c|c|c|c|}
\hline \multirow{3}{*}{ Дата } & \multicolumn{6}{|c|}{$\begin{array}{l}\text { Ранговый коэффициент корреляции r } \text {, д. ед., связи значений } \boldsymbol{L} \lambda \text { и показателей морфологии } \\
\text { активных оползневых тел. }\end{array}$} \\
\hline & \multicolumn{2}{|c|}{ Параметры склона } & \multicolumn{4}{|c|}{ Характеристики активных оползневых тел } \\
\hline & Длина L, м & $\begin{array}{l}\text { Высота } \\
\text { Н, м }\end{array}$ & $\begin{array}{l}\text { Длина, } \\
1, \text { м }\end{array}$ & Ширина, b, м & Площадь, s, м² & $\begin{array}{l}\text { Высота стенки отрыва, } \\
\text { h, м }\end{array}$ \\
\hline 18.05 .1988 & $-0,40$ & 0,247 & - & - & - & $-0,219$ \\
\hline 18.02 .1990 & $-0,266$ & - & - & - & - & $-0,224$ \\
\hline 20.02 .2003 & $-0,219$ & - & - & $-0,175$ & - & - \\
\hline 23.04 .2003 & - & 0,285 & $-0,263$ & $-0,391$ & $-0,384$ & - \\
\hline 26.06 .2003 & - & & $-0,202$ & $-0,281$ & $-0,27$ & - \\
\hline 26.06 .2009 & - & $-0,249$ & $-0,405$ & $-0,28$ & $-0,387$ & - \\
\hline 22.01 .2010 & $-0,221$ & - & - & - & - & - \\
\hline 25.03 .2010 & - & - & - & $-0,202$ & $-0,173$ & - \\
\hline 24.01 .2011 & - & - & - & $-0,203$ & $-0,189$ & - \\
\hline
\end{tabular}

Примечания к табл. 1-3:

1. В таблицах приведены статистически значимые величины коэффициента ранговой корреляции и уравнения регрессии.

2. (-) - отсутствует значимый коэффициент корреляции.

влияния солнечной активности (даты 23.04.2003, 26.06.2009). Достоверных уравнений регрессии, которые можно было бы использовать для прогноза оползневой активности, получить не удалось (см. табл. 2).

Методом группового учета аргументов получены многочисленные модели зависимости значений $\boldsymbol{L} \boldsymbol{\lambda}$ от указанных переменных. Анализ уравнений показывает, что они нелинейны, вклады детермини- ных наиболее часто выступают высота склона $\boldsymbol{H}, \mathbf{\text { м, }}$ ширина оползневого тела $\boldsymbol{b}, \mathbf{M}$, номер точки, длина оползневого тела $\boldsymbol{L}$, м и высота стенки срыва $\boldsymbol{h}, \mathbf{M}$.

Анализ показал, что в 2009 году в данных присутствуют точки с экстремально высокими значениями приходящего на сенсор излучения, в которых значение $\boldsymbol{L} \boldsymbol{\lambda}$ составило $0,49 \frac{\mathrm{B}}{\mathrm{M}^{2} \cdot \text { ster } \mu \mathrm{M}}$. Точки локализованы, расположены в границах двух эрозионных бассейнов 
Модели регрессии излучения на сенсоре $L \lambda$ и показателей морфологии оползневых тел.

\begin{tabular}{|l|l|l|l|}
\hline Дата & Уравнения регрессии & $\begin{array}{l}\text { Коэффициент детерминации } \mathrm{R}, \\
\text { д.ед. }\end{array}$ & $\begin{array}{l}\text { Уточненный } \\
\text { коэффициентАR }{ }^{2}, \text { д. ед. }\end{array}$ \\
\hline 18.05 .1988$, & $\boldsymbol{L} \boldsymbol{\lambda}=0,113-0,000001 \mathrm{~L}+0,000148 \mathrm{H}$ & 0,50 & 0,21 \\
\hline 18.02 .1990 & $\boldsymbol{L} \boldsymbol{\lambda}=0,106-0,000001+0,0000361$ & 0,41 & 0,17 \\
\hline 26.06 .2009 & $\boldsymbol{L} \boldsymbol{\lambda}=0,138+0,0000001 \mathrm{~L}$ & 0,28 & 0,036 \\
\hline 22.01 .2010 & $\boldsymbol{L} \boldsymbol{\lambda}=0,102-0,000001 \mathrm{~L}$ & 0,31 & 0,051 \\
\hline 25.03 .2010 & $\boldsymbol{L} \boldsymbol{\lambda}=0,107-0,000001 \mathrm{~L}-0,000129 \mathrm{H}$ & 0,391 & 0,112 \\
\hline
\end{tabular}

Примечания к табл. 2: L - длина, м; Н - высота, м.

балок Красноповстанческая и Запорожская, в которых оползневые процессы развивались (на застроенной территории) наиболее активно. Выборка была раз- отрыва, ширина оползневого тела и высота склона. На участках с экстремальными значениями $\boldsymbol{L} \boldsymbol{\lambda}$ параметрами являются, в основном, длина оползневого

Таблица 3

Результаты индуктивного моделирования зависимости L $\lambda$ от параметров оползневых тел

\begin{tabular}{|c|l|l|l|l|l|l|l|l|}
\hline \multirow{3}{*}{ Дата } & \multicolumn{7}{|c|}{ Параметры склона и оползневого тела } \\
\cline { 2 - 9 } & Переменная & $\begin{array}{l}\text { Номер } \\
\text { точки № }\end{array}$ & $\begin{array}{l}\text { Длина } \\
\text { оползневого } \\
\text { тела L, м }\end{array}$ & $\begin{array}{l}\text { Высота } \\
\mathrm{H}, \mathrm{m}\end{array}$ & $\begin{array}{l}\text { Длина, } \\
1, \mathrm{~m}\end{array}$ & $\begin{array}{l}\text { Ширина, } \\
\mathrm{b}, \mathrm{m}\end{array}$ & $\begin{array}{l}\text { Площадь, } \\
\mathrm{s}, \mathrm{M}^{2}\end{array}$ & $\begin{array}{l}\text { Высота } \\
\text { стенки } \\
\text { отрыва, } \\
\mathrm{h}, \mathrm{m}\end{array}$ \\
\cline { 2 - 10 } & Аргумент модели & $\mathrm{x}_{1}$ & $\mathrm{x}_{2}$ & $\mathrm{x}_{3}$ & $\mathrm{x}_{4}$ & $\mathrm{x}_{5}$ & $\mathrm{x}_{6}$ & $\mathrm{x}_{7}$ \\
\hline 18.02 .1990 & $-6,764$ & - & $-2,22$ & - & 0,341 & & 1,066 \\
\hline 20.02 .2003 & $-0,024$ & $-0,198$ & 0,071 & 0,122 & - & $-0,019$ & - \\
\hline 23.04 .2003 & 0,717 & - & 0,139 & 0,044 & 0,025 & - & 0,014 \\
\hline 26.06 .2009 & 0,229 & - & $-0,051$ & $-0,008$ & $-0,338$ & 0,217 & $-0,008$ \\
\hline 22.01 .2010 & - & $-0,272$ & 0,04 & 0,015 & $-0,051$ & 0,037 & $-0,024$ \\
\hline
\end{tabular}

Примечания:

1.№ - Порядковый номерточки.

2.Приведены величины детерминированной составляющей индуктивных моделей зависимости $L \lambda$ от параметров склона и оползневого тела.

3.В таблице приведены сведения о всех полученных моделях.

делена на две части, содержащие экстремальные и «фоновые» значения. Методом группового учета аргументов получены модели зависимости излучения на сенсоре $\boldsymbol{L} \boldsymbol{\lambda}$ от параметров склона и оползневого тела для обеих выборок (табл. 4).

Для участков с фоновыми значениями $L \lambda$ наиболее четко выраженными параметрами детерминированной компоненты выступают высота стенки тела и протяженность склона.

Выполнен прогноз значений активности оползневого процесса в каждой из экстремальных точек. Стандартизованные значения излучения поверхностив двух точках возросли до значений 1,589 и 1,684 д.ед., а во всех точках уменьшились. Можно сделать вывод, что в целом вероятно снижение оползневой активности в границах

Коэффициенты при детерминированных членах модели влияния параметров склона и оползневого тела на их спектральные показатели (2009 г.)

\begin{tabular}{|l|l|l|l|l|l|l|l|}
\hline $\begin{array}{l}\text { Выборочные } \\
\text { данные }\end{array}$ & $\mathrm{x}_{1}$ & $\mathrm{x}_{2}$ & $\mathrm{x}_{3}$ & $\mathrm{x}_{4}$ & $\mathrm{x}_{5}$ & $\mathrm{x}_{6}$ & $\mathrm{x}_{7}$ \\
\hline $\begin{array}{l}\text { Экстремальные } \\
\text { значения }\end{array}$ & $-528,6$ & 123,74 & 1,36 & $-191,72$ & - & - & - \\
\hline $\begin{array}{l}\text { Фоновые } \\
\text { значения }\end{array}$ & 0,005 & - & 0,005 & - & - & $-0,03$ & 0,03 \\
\hline
\end{tabular}

Примечание к таблице 4:

$\mathrm{x}_{1}$ - номер точки; $\mathrm{x}_{2}$ - протяженность склона; $\mathrm{x}_{3}$ - высота склона; $\mathrm{x}_{4}$ - длина оползневого тела; $\mathrm{x}_{5}$ - ширина оползневого тела; $\mathrm{x}_{6}$ - площадь; $\mathrm{x}_{7}$ - высота стенки отрыва. 
изученной территории, за исключением отдельных опасных участков.

Выводы. В результате выполненных исследований подтверждена связь между развитием оползневых явлений на территории г. Днепропетровск и $11-$ летними вариациями солнечной активности. Применение методов индуктивного моделирования (метода группового учета аргументов) позволяет выполнить прогноз активизации оползневого процесса для оползневых тел, находящихся в фазе активного развития, с использованием доступных материалов дистанционного зондирования.

\section{Библиографические ссылки}

Caers J., 2011. Modeling Uncertainty in the Earth Sciences - John Wiley \& Sons Ltd. - 224 p.

cite web |url= http://aviris.gl.fcen.uba.ar/Curso SR/ biblio sr/ENVI userguid.pdf=ENVI user`s guide. ENVI version $4.1 /$ publisher $=$ Open Publishing $\mid$ date $=30$ квітня 2005 |work=Encyclopedia of Things |language=англ.

cite web |url=http://glovis.usgs.gov=Intreaktivnyj WEB-interfejs poluchenija dannyh distancionnogo zondirovanija $\mathrm{s}$ resursa Geologicheskoj Sluzhby Soedinjonnyh Shtatov

Das, I. Ch. Spatial Statistical modelling for assessing landslides hazards vulnerability. Dissertation PhD. - University of Twenty, The Netherland.-171 p.

Emel'janova, E.P., 1971. Sravnitel'ny metod ocenki ustojchivosti sklonov I prognoza opolznej [Comparative evaluation method of slope stability and landslide forecast]. M: Nedra. (in Russian).

Groisman, C.P., Lyalko, V., 2012. Earth Systems Change over Eastern Europe. K. :Akadem periodyka.

Gulakjan, K.A., Kjuncel', V.V., Postoev, G.P., 1977. Prognozirovanie opolznevyh processov [Prediction of landslides]. M.: Nedra. (in Russian)

Koposov, E.V., 2012. Metodologicheskoe obespecheniej ekologicheskoj bezopasnosti stroitel'stva na urbanizirovannyh territorijah, podverzhennyh vozdejstviju opolznevyh processov. [Methodological maintenance of ecological safety of construction in urban areas exposed to landslides]. Bulletin MGSU, 3, 138 - 144. (in Russian)

Kuz'menko, E.D., Shkica, L.E., 2007. Geotehnogenna bezpeka rekreacijno-turistichnih teritorij Ukraini. [Technogenic security of recreational and tourist areas in Ukraine.] Building and technological safety', issue 21, 109- 113. (in Ukrainian)

Mokrickaja, T. P., Korjashkina, L. S., 2013. Faktory i modeli degradacii prosadochnosti [Factors and model degradation subsidence]. Visnik Nacional'nogo girnichogo universitetu, 4, 5 - 12 (in Russian).

Montoya-Montes, I., Rodríguez-Santalla, M.J., Sánchez-García, J., Alcántara-Carriós, Martín-Velázquez, D., GómezOrtiz, T., Martín-Crespo, 2012.Mapping of landslide susceptibility of coastal cliffs: the Mont-Roig del Camp case study/ Geologica Acta, Vol. 10, 4, December, 439 - 455. doi: 10.1344/105.000001776

Pardeshi, S.D., Autade, S.E., Pardeshi, S.S., 2013.Springer Plus Landslide hazard assessment: recent trends and techniques. 2: 523. doi:10.1186/2193-1801-2-523

Patel, N., Sahoo, G., Singh, A. K., 2013. Jmage Classification for Different Land Use and Land Covers UsingFuzzy Logic for the Improvement of Accuracies. Journal of Agricultural Science; 5, 3; 2013, $278-283$.

Shljahova, L.A, Povh, V.I., 2010.Ocenka ugroz opolznevyh processov na territorii JuFO po dannym DZZ [Assessment of threats of landslide processes on the territory of JuFO according to DZZ] Eighth All-Russia open annual conference «Modern problems of remote sensing of the Earth from space.”. Moskow, IKI RAN. (in Russian) 